\title{
On the stability of the Motzkin representation of closed convex sets
}

\author{
M.A. Goberna \\ Dep. of Statistics and Operations Research, Universidad de Alicante, Spain, \\ E-mail:mgoberna@ua.es.
}

\section{I. Todorov}

Dep. Actuary and Mathematics, Universidad de las Américas, Cholula, Puebla, Mexico, E-mail: maxim.todorov@udlap.mx. On leave IMI-BAS, Sofia, Bulgaria.

\begin{abstract}
A set is called Motzkin decomposable when it can be expressed as the Minkowski sum of a compact convex set with a closed convex cone. This paper analyzes the continuity properties of the set-valued mapping associating to each couple $(C, D)$ formed by a compact convex set $C$ and a closed convex cone $D$ its Minkowski sum $C+D$. The continuity properties of other related mappings are also analyzed.
\end{abstract}

Key words: Motzkin decomposition, Minkowski sum, stability

\section{Introduction}

We say that a nonempty set $F \subset \mathbb{R}^{n}$ is decomposable in Motzkin's sense (M-decomposable in short) if there exist a compact convex set $C$ and a closed convex cone $D$ such that $F=C+D$. Then we say that $C+D$ is a Motzkin representation (or decomposition) of $F$ with compact and conic components $C$ and $D$, respectively. Any M-decomposable set $F$ has a unique conic component $D=0^{+} F$ (the recession cone of $F$ ) and $F=F+\left\{0_{n}\right\}$ is the unique Motzkin decomposition of $F$ whenever $F$ is bounded. The classical Motzkin Theorem [17] asserts that any polyhedral convex set is M-decomposable. This class of closed convex sets has been characterized in different ways in [7], [8] and [9]. For instance, a closed convex set $F \subset \mathbb{R}^{n}$ is M-decomposable iff $F \cap(\operatorname{lin} F)^{\perp}$ is M-decomposable iff the Pareto-like set of $F$,

$$
M(F):=\left\{x \in F \cap(\operatorname{lin} F)^{\perp}:\left(x-\left(0^{+} F\right) \cap(\operatorname{lin} F)^{\perp}\right) \cap F=\{x\}\right\},
$$


is bounded (here $\operatorname{lin} F:=\left(0^{+} F\right) \cap\left(-0^{+} F\right)$ denotes the lineality space of $F$ and $(\operatorname{lin} F)^{\perp}$ its orthogonal complement). In that case, [7, Theorem 19] shows that

$$
F=\operatorname{cl} \operatorname{conv} M(F)+0^{+} F=\operatorname{cl} M(F)+0^{+} F,
$$

although the last equation is not explicit in the statement). If $F$ contains no line, $M(F)=\left\{x \in F:\left(x-0^{+} F\right) \cap F=\{x\}\right\}$ is the efficient set of $F$ relative to the cone $0^{+} F$ and $C(F):=\operatorname{cl}$ conv $M(F)$ is the smallest compact component of $F$ (which does not exist when $F$ contains lines).

M-decomposable sets with uncertain compact component arise, for instance, in Data Envelopment Analysis (DEA), whose purpose is the comparison of the efficiency of a set of decision making units (e.g., firms, factories, branches or schools) or technologies in order to obtain certain outputs from the available inputs. When the number of decision making units (DMUs) to be compared is $p \in \mathbb{N}$, the efficiency ratios are usually computed via Linear Programming from a set of the form $C+\mathbb{R}_{+}^{n}$, where $C$ is the convex hull of $\left\{x_{1}, \ldots, x_{p}\right\} \subset \mathbb{R}^{n}$ and each $x_{j}$ depends on the inputs and outputs of the $j$-th DMU (e.g., see [5]). Analogously, in the case of chemical processes which are controlled by means of certain parameters (pressure, temperature, concentrations, etc.), the efficiency ratios of the virtual technologies are computed via Linear SemiInfinite (or Bilevel) Programming ([13]) from a set of the form $C+\mathbb{R}_{+}^{n}$ where $C$ is the convex hull of certain infinite compact set $X \subset \mathbb{R}^{n}$. In all practical applications, the set $\left\{x_{1}, \ldots, x_{p}\right\}$ (or its infinite counterpart $X$ ) is uncertain, i.e., the compact convex set $C$ is subject to perturbations whereas the closed convex cone $D$ remains fixed, so that the efficiency ratios are also uncertain and their stability behavior depend on the stability behavior of $C+\mathbb{R}_{+}^{n}$ under sufficiently small perturbations of $C$.

The main objective of this paper is the study of the stability properties of the sum of a compact convex set with a closed convex cone when one of these two sets, or both, are subject to small perturbations that preserve the mentioned properties. This problem can be seen as a particular case of the following one: studying the stability of the feasible set for the different types of representations of closed convex sets. In fact, representing a given closed convex set $F \subset \mathbb{R}^{n}$ consists of choosing an element $\theta$ (called nominal parameter) in certain set $\Theta$ (called parameter space) whose elements are the results of all admissible perturbations of $\theta$ (due, e.g., to the inaccuracy of the data). It is assumed the existence of a set-valued mapping $\mathcal{F}: \Theta \rightrightarrows \mathbb{R}^{n}$, called feasible set mapping, associating to each perturbation of the nominal data $\theta$ the corresponding perturbation of $F$. Obviously, we must have, in particular, $\mathcal{F}(\theta)=F$. The domain of $\mathcal{F}$ is $\operatorname{dom} \mathcal{F}:=\left\{\theta_{1} \in \Theta: \mathcal{F}\left(\theta_{1}\right) \neq \emptyset\right\}$. A closed convex set $F_{1}$ has a unique representation in $\Theta$ when $\mathcal{F}^{-1}\left(F_{1}\right)$ is singleton. The question to be answered, from the stability perspective, is whether the effect on the feasible set of small perturbations of the data are necessarily small too, so that we also assume that $\Theta$ is endowed with some topology. The stability 
results on $\mathcal{F}$ either characterize the topological interior of $\operatorname{dom} \mathcal{F}$ (i.e., the elements of $\Theta$ that represent nonempty closed convex sets under sufficiently small perturbations) or provide conditions for the continuity (in some sense) of $\mathcal{F}$ at $\theta \in \operatorname{dom} \mathcal{F}$. The completeness of some neighborhood of $\theta$ is a desirable feature of the selected type of representation as far as this property connects the continuity properties of $\mathcal{F}$ at $\theta$ with the metric regularity of $\mathcal{F}^{-1}$ at $(x, \theta)$, with $x \in \mathcal{F}(\theta)$. Now we describe briefly three types of representations of closed convex sets, namely: linear, conic and Motzkin representations (the one we are interested in).

Let $F$ be the solution set of the linear system $\theta=\left\{a_{t}^{\prime} x \geq b_{t}, t \in T\right\}$ (the given linear representation of $F$ ). Then $\Theta$ is the class of all linear systems obtained by perturbing the coefficients of $\theta$ (i.e., the functions $a: T \rightarrow \mathbb{R}^{n}$ and $b: T \rightarrow \mathbb{R}$ ) maintaining the number of variables and constraints, $n$ and $T$. In other words, $\Theta=\left(\mathbb{R}^{n} \times \mathbb{R}\right)^{T}$ and, given $\theta_{1}=\left\{\left(a_{t}^{1}\right)^{\prime} x \geq b_{t}^{1}, t \in T\right\} \in \Theta$,

$$
\mathcal{F}\left(\theta_{1}\right):=\left\{x \in \mathbb{R}^{n}:\left(a_{t}^{1}\right)^{\prime} x \geq b_{t}^{1}, t \in T\right\}
$$

Observe that, given an arbitrary set of positive numbers $\left\{\lambda_{t}, t \in T\right\}, \theta_{1}=$ $\left\{\lambda_{t} a_{t}^{\prime} x \geq \lambda_{t} b_{t}, t \in T\right\}$ is another linear representation of $F$ in $\Theta$, so that there are infinitely many linear representations of $F$. The most common way to measure the size of the perturbations appeals to the metric of the uniform convergence introduced in [12]: given $\theta_{i}=\left\{\left(a_{t}^{i}\right)^{\prime} x \geq b_{t}^{i}, t \in T\right\} \in \Theta, i=1,2$,

$$
\rho\left(\theta_{1}, \theta_{2}\right):=\sup _{t \in T}\left\|\left(\begin{array}{c}
a_{t}^{1} \\
b_{t}^{1}
\end{array}\right)-\left(\begin{array}{c}
a_{t}^{2} \\
b_{t}^{2}
\end{array}\right)\right\|_{\infty},
$$

where $\|\cdot\|_{\infty}$ stands for the Chebyshev norm in $\mathbb{R}^{n+1}$ (actually $\rho$ is an infinitevalued metric iff $T$ is infinite). The neighborhood of $\theta$ formed by the finite perturbations of the nominal parameter $\theta,\left\{\theta_{1} \in \Theta: \rho\left(\theta_{1}, \theta\right)<+\infty\right\}$, is a complete metric space.

In the conic representation perspective, the nominal parameter $\theta$ is a given closed convex cone in $\mathbb{R}^{n+1}$ such that $\left(0_{n},-1\right) \in \theta$ and

$$
F=\left\{x \in \mathbb{R}^{n}: a^{\prime} x \geq b \forall(a, b) \in \theta\right\},
$$

with $F \neq \emptyset$ if and only if $\left(0_{n}, 1\right) \notin \theta$. In that case, $F$ is bounded if and only if $\left(0_{n}, 1\right)$ is an interior point of $\theta([10])$. It is worth observing that, if $F \neq \emptyset, \theta$ coincides with the reference cone of $F$, i.e.,

$$
\theta=\left\{(a, b) \in \mathbb{R}^{n+1}: a^{\prime} x \geq b \forall x \in F\right\},
$$

so that there is a unique conic representation for every nonempty closed convex set. Now $\Theta$ is formed by the class of all closed convex cones in $\mathbb{R}^{n+1}$ containing 
the vector $\left(0_{n},-1\right)$ and, given $\theta_{1} \in \Theta$,

$$
\mathcal{F}\left(\theta_{1}\right):=\left\{x \in \mathbb{R}^{n}: a^{\prime} x \geq b \forall(a, b) \in \theta_{1}\right\} .
$$

Obviously, the Hausdorff metric $d_{H}$ in $\mathbb{R}^{n+1}$ defined in (5) is an inconvenient measure for the size of the perturbations of $\theta$ because $d_{H}\left(\theta_{1}, \theta\right)=+\infty$ for all $\theta_{1} \in \Theta$ such that $\theta_{1} \neq \theta$. One of the ways to avoid this drawback is to replace $d_{H}\left(\theta_{1}, \theta\right)$ by $d_{H}\left(\psi\left(\theta_{1}\right), \psi(\theta)\right)$, where $\psi$ is the truncation mapping associating to each closed convex cone its intersection with the unit closed ball (it could be another compact neighborhood of the origin), i.e., $\psi\left(\theta_{1}\right):=\theta_{1} \cap B_{n+1}$, $\theta_{1} \in \Theta$. Thus, a suitable metric on $\Theta$ is

$$
\rho\left(\theta_{1}, \theta_{2}\right):=d_{H}\left(\psi\left(\theta_{1}\right), \psi\left(\theta_{2}\right)\right)=d_{H}\left(\theta_{1} \cap B_{n+1}, \theta_{2} \cap B_{n+1}\right) \leq 1, \theta_{1}, \theta_{2} \in \Theta .
$$

A simple modification of Lemma 4 shows that $\langle\Theta, \rho\rangle$ is complete.

This paper is focussed on the stability of the feasible set mapping for Motzkin representations of those closed convex sets which are M-decomposable, i.e., the nominal parameter is a couple $\theta=(C, D)$ such that $C$ is a compact convex set, $D$ is a closed convex cone, and $F=C+D$, whereas $\Theta$ is the cartesian product of the space of nonempty compact convex sets $\Delta_{1}$ and the space of closed convex cones $\Delta_{2}$, endowed with a suitable metric (the product of Hausdorff metrics, denoted by $d_{H} \times d_{H}$, is an inconvenient metric for $\Theta$ because $\left(d_{H} \times d_{H}\right)\left(\theta_{1}, \theta\right)=+\infty$ if $\theta_{1}=\left(C_{1}, D_{1}\right) \in \Theta$ satisfies $D_{1} \neq D$, i.e., any perturbation of the conic component has infinite size). So, we consider the product of $\left\langle\Delta_{1}, d_{H}\right\rangle$ and $\left\langle\Delta_{2}, d_{H} \circ(\psi, \psi)\right\rangle$, say $\langle\Theta, \rho\rangle$. In other words, the distance between $\theta_{1}=\left(C_{1}, D_{1}\right)$ and $\theta_{2}=\left(C_{2}, D_{2}\right)$ is

$$
\rho\left(\theta_{1}, \theta_{2}\right):=\max \left\{d_{H}\left(C_{1}, C_{2}\right), d_{H}\left(D_{1} \cap B_{n}, D_{2} \cap B_{n}\right)\right\}
$$

so that the topology induced by $\rho$ on $\Theta$ is the product of the topologies induced by the Hausdorff metric (5) on $\Delta_{1}$ and the Hausdorff-like metric (3) on $\Delta_{2}$ (the induced topology on $\Delta_{2}$ coincides with the so-called bounded Hausdorff topology; see, e.g., [14]), respectively. Our feasible set mapping is $\mathcal{F}: \Theta \rightrightarrows \mathbb{R}^{n}$ such that

$$
\mathcal{F}\left(\theta_{1}\right):=C_{1}+D_{1},
$$

for any $\theta_{1}=\left(C_{1}, D_{1}\right) \in \Theta$. Observe that the Motzkin representation of $F=$ $C+D$ is not unique when $D \neq\left\{0_{n}\right\}$ (i.e., when $F$ is unbounded) because $\mathcal{F}(\operatorname{conv}\{C \cup(C+\lambda d)\}, D)=\mathcal{F}(C, D)$ for every $d \in D \backslash\left\{0_{n}\right\}$ and $\lambda>0$. Obviously, the problem of analyzing the stability of $\mathcal{F}$ can also be seen as a particular case of the more general one of studying the continuity properties of the sum of closed convex sets.

The secondary purpose of this paper is the stability analysis of the set-valued mappings $\mathcal{M}, \mathcal{C}:(\Theta, \rho) \rightrightarrows \mathbb{R}^{n}$ such that $\mathcal{M}\left(\theta_{1}\right)=M\left(\mathcal{F}\left(\theta_{1}\right)\right)$ and $\mathcal{C}\left(\theta_{1}\right)=$ $C\left(\mathcal{F}\left(\theta_{1}\right)\right):=\operatorname{cl} \operatorname{conv} \mathcal{M}\left(\theta_{1}\right)$ for all $\theta_{1} \in \Theta$. 
The paper is organized as follows. Section 2 contains the necessary notation, antecedents, and auxiliary results to be used later. Section 3 proves the completeness of the parameter spaces, Section 4 analyzes the stability of $\mathcal{F}$ and Section 5 studies the stability of $\mathcal{M}$ and $\mathcal{C}$.

\section{Preliminaries}

Throughout the paper we use the following notation and concepts.

Let $X$ be a metrizable space. For any $A \subset X$, int $A, \operatorname{cl} A$, and bd $A$ denote the interior, the closure, and the boundary of $A$, respectively. We denote by $C L(X)$ the class of all nonempty closed subsets of $X$ and by $2^{X}$ the class of all closed subsets of $X$, i.e., $2^{X}:=C L(X) \cup\{\emptyset\}$. Let us recall the continuity concepts for set-valued mappings we use in the sequel. Let $\mathcal{N}: X \rightrightarrows \mathbb{R}^{n}$.

$\mathcal{N}$ is closed at $x$ if for any $y \in \mathbb{R}^{n}$ and any two sequences, $\left\{x_{k}\right\} \subset X$ and $\left\{y_{k}\right\} \subset \mathbb{R}^{n}$ such that $\lim _{k} x_{k}=x, y_{k} \in \mathcal{N}\left(x_{k}\right), k=1,2, \ldots$, and $\lim _{k} y_{k}=y$, one gets $\bar{y} \in \mathcal{N}(x)$.

$\mathcal{N}$ is lower semicontinuous in Berge-Kuratowski sense (lsc) at $x$ if for each open set $U$ such that $U \cap \mathcal{N}(x) \neq \emptyset$ there exists an open set $V, x \in V \subset X$, such that $U \cap \mathcal{N}\left(x_{1}\right) \neq \emptyset$ for every $x_{1} \in V$.

$\mathcal{N}$ is upper semicontinuous in Berge-Kuratowski sense (usc) at $x$ if for each open set $U$ such that $\mathcal{N}(x) \subset U$ there exists an open set $V, x \in V \subset X$, such that $\mathcal{N}\left(x_{1}\right) \subset U$ for every $x_{1} \in V$. This stability property is considered too strong in most frameworks (see, e.g., [18]).

Finally, we say that $\mathcal{N}$ is closed (lsc, usc) on $X$ when it is closed (lsc, usc) at $x$ for all $x \in X$.

Given $A \subset X=\mathbb{R}^{p}$, we denote by $\operatorname{rint} A$, conv $A$, and cone $A=\mathbb{R}_{+}$conv $A$, the relative interior, the relative boundary, the convex hull of $A$, and the convex conical hull of $A$, respectively. The scalar product of $x, y \in \mathbb{R}^{p}$ is denoted either by $x^{\prime} y$ or by $\langle x, y\rangle$, the Euclidean norm of $x$ by $\|x\|$, the canonical basis by $\left\{e_{1}, \ldots, e_{p}\right\}$, the zero vector by $0_{p}$, the closed unit ball by $B_{p}$, and the Hausdorff distance between two closed sets $A, B$ by

$$
d_{H}(A, B)=\inf \left\{\eta \in \mathbb{R}_{+}: A \subset B+\eta B_{p} \text { and } B \subset+\eta B_{p}\right\} .
$$

Let $\left\{A_{k}\right\}$ be a sequence of nonempty sets in $\mathbb{R}^{p}$. We denote by $\liminf { }_{k} A_{k}$ $\left(\lim \sup _{k} A_{k}\right)$ the set formed by all the possible limits (cluster points, respectively) of sequences $\left\{x_{k}\right\}$ such that $x_{k} \in A_{k}$ for all $k \in \mathbb{N}$ (we usually write $\lim _{k} x_{k}=x$, or even $x_{k} \rightarrow x$, instead of $\lim _{k \rightarrow \infty} x_{k}=x$ ). When these two limit 
sets are non-empty and coincide, then it is said that $\left\{A_{k}\right\}$ converges in the Painlevé-Kuratowski sense to the set

$$
\lim _{k} A_{k}:=\liminf _{k} A_{k}=\limsup _{k} A_{k} .
$$

Then we write $A_{k} \rightarrow^{P K} \lim _{k} A_{k}$. If $\left\{A_{k}\right\}$ is a sequence of closed sets in $\mathbb{R}^{p}$ such that $\lim _{k} d_{H}\left(A_{k}, A\right)=0$ (in short $A_{k} \rightarrow^{H} A$ ), then $A_{k} \rightarrow^{P K} A$ and the converse statement holds when there exists $\mu>0$ such that $A, A_{k} \subset \mu B_{p}$ for all $k \in \mathbb{N}([18])$.

Now we summarize the antecedents on the stability of the feasible set for linear and conic representations.

Concerning linear representations, it is easy to prove that $\langle\Theta, \rho\rangle$, with $\rho$ defined as in (2), is a complete metric space. Moreover, it is known (see, e.g., [11]) that $\mathcal{F}$ is closed whereas $\mathcal{F}$ is lsc at $\theta=\left\{a_{t}^{\prime} x \geq b_{t}, t \in T\right\} \in \operatorname{dom} \mathcal{F}$ iff $\theta \in \operatorname{int} \operatorname{dom} \mathcal{F}$ iff $0_{n+1} \notin \operatorname{clconv}\left\{\left(a_{t}, b_{t}\right), t \in T\right\}$ (a condition involving the data). The usc property of $\mathcal{F}$ was characterized later, in [3], by means of a condition on the set $\left\{\left(a_{t}, b_{t}\right), t \in T\right\}$ which is usually difficult to be checked. A sufficient condition for the usc property of $\mathcal{F}$ at $\theta$ is that $\mathcal{F}(\theta)$ is either a compact set or the whole space $\mathbb{R}^{n}$. When $T$ is a compact Hausdorff topological space and $\Theta$ is formed by the linear inequality systems in $\mathbb{R}^{n}$ whose coefficients are continuous functions of the index $t$ on $T$ (which trivially holds when $T$ is finite by considering the discrete topology on $T$ ), then $\Theta$ is a Banach space, $\mathcal{F}$ is closed, the lsc property of $\mathcal{F}$ at $\theta \in \operatorname{dom} \mathcal{F}$ is characterized as before, and $\mathcal{F}$ is usc at $\theta \in \operatorname{dom} \mathcal{F}$ iff $\mathcal{F}(\theta)$ is either a compact set or the whole space $\mathbb{R}^{n}$ $([2],[6])$.

Concerning the stability of the feasible set mapping for conic representations, it is just known that $\operatorname{dom} \mathcal{F}$ is open and bd $\operatorname{dom} \mathcal{F}$ is the class of inconsistent linear systems whose finite subsystems are consistent ([16]). Thus $\Theta \backslash \operatorname{cldom} \mathcal{F}$ is formed by the strongly inconsistent linear systems (those systems containing some inconsistent subsystem). The continuity properties of $\mathcal{F}$ in this framework have not yet been explored.

The set-valued mapping $\mathcal{M}:(\Theta, \rho) \rightrightarrows \mathbb{R}^{n}$ is related with the efficient set mapping in multiobjective optimization, whose stability properties have been widely analyzed in the literature for linear (and nonlinear) representations of the feasible set $F$ (see, e.g., [19], [20], [4], and references therein). F.i., in the case of linear representations, under suitable conditions, generic lower semicontinuity of the mapping $\mathcal{M}$ has been proven in ([20]). In our setting we have to exploit the special structure of the vector optimization problems, coming from the ordering cone $D=0^{+} F$, which, f.i., keeps the sets $M(F)$ always bounded, a fact which is not true in general.

Now we consider the parameter space of the Motzkin representations, $\langle\Theta, \rho\rangle$, 
where $\rho$ is the product of $d_{H}$ and $d_{H} \circ(\psi, \psi)$, with $\psi\left(D_{1}\right)=D_{1} \cap B_{n}$ for any closed convex cone $D_{1}$. Next we apply two known results on the sum of closed sets in $\mathbb{R}^{n}$ to obtain consequences for the stability of feasible set mapping $\mathcal{F}$ in terms of the continuity of the associated single-valued mapping $\widetilde{\mathcal{F}}: \Theta \rightarrow C L\left(\mathbb{R}^{n}\right)$ such that $\widetilde{\mathcal{F}}\left(C_{1}, D_{1}\right):=\left\{C_{1}+D_{1}\right\}$. Unfortunately, in these results $\mathcal{F}$ ranges on $\left\langle\Theta, d_{H} \times d_{H}\right\rangle$, where $\Theta$ is the union of disjoint open and closed sets which are formed by parameters sharing the same compact component, in such a way that the distance between two parameters is finite iff they belong to the same element of the partition.

Consider the set-valued mapping $\mathcal{S}: 2^{\mathbb{R}^{n}} \times 2^{\mathbb{R}^{n}} \rightrightarrows \mathbb{R}^{n}$ such that $\mathcal{S}(A, B)=$ $\operatorname{cl}(A+B)$ for each pair $A, B$ of closed sets in $\mathbb{R}^{n}$, whose associated singlevalued mapping is $\widetilde{\mathcal{S}}: 2^{\mathbb{R}^{n}} \times 2^{\mathbb{R}^{n}} \rightarrow 2^{\mathbb{R}^{n}}$ such that $\widetilde{\mathcal{S}}(A, B)=\{\operatorname{cl}(A+B)\}$. It is known that its restriction $\widetilde{\mathcal{S}}:\left\langle C L\left(\mathbb{R}^{n}\right) \times C L\left(\mathbb{R}^{n}\right), d_{H} \times d_{H}\right\rangle \rightarrow\left\langle C L\left(\mathbb{R}^{n}\right), d_{H}\right\rangle$ is continuous (see, e.g., [1, Exercise 3.2.12]). Since $\widetilde{\mathcal{S}}=\widetilde{\mathcal{F}}$ on $\Theta$, we conclude that $\widetilde{\mathcal{F}}:\left\langle\Theta, d_{H} \times d_{H}\right\rangle \rightarrow\left\langle C L\left(\mathbb{R}^{n}\right), d_{H}\right\rangle$ is continuous too.

On the other hand, since $\mathcal{S}$ has closed images, $\widetilde{\mathcal{S}}$ is continuous relative to the Vietoris topology on $C L\left(\mathbb{R}^{n}\right) \times C L\left(\mathbb{R}^{n}\right)$ iff $\mathcal{S}: 2^{\mathbb{R}^{n}} \times 2^{\mathbb{R}^{n}} \rightrightarrows \mathbb{R}^{n}$ is both lsc and usc ([1, Theorem 6.2.9]). Thus, the restriction of $\mathcal{S}$ to $\Theta, \mathcal{F}:\left\langle\Theta, d_{H} \times d_{H}\right\rangle \rightrightarrows$ $\mathbb{R}^{n}$, is lsc and usc iff $\widetilde{\mathcal{F}}$ is continuous relative to the Vietoris topology on $\Theta$.

\section{Completeness of the parameter space}

From now on we consider the parameter space $\langle\Theta, \rho\rangle$, with $\rho$ defined as in (4). The next example, to be used later, shows the existence of sequences of M-decomposable sets that converge in Painlevé-Kuratowski sense to another M-decomposable set whereas their respective Motzkin representations may converge or not, in the metric $\rho$.

Example 1 Consider the sequence of $M$-decomposable sets

$$
F_{k}=\left\{x \in \mathbb{R}^{2}: x_{1} \geq 0, x_{2} \geq 0,-\frac{1}{k} x_{1}+x_{2} \geq-\frac{1}{k}\right\}, k \in \mathbb{N} .
$$

It can be realized that

$$
F_{k} \rightarrow P K\left\{x \in \mathbb{R}^{2}: x_{1} \geq 0, x_{2} \geq 0\right\}=F
$$

(another M-decomposable set) even though $\left\{F_{k}\right\}$ is not a Cauchy sequence relative to $\rho$. Observe that the smallest compact component of $F_{k}$ is constant, $C_{k}=[0,1] \times\{0\}$, with $C_{k} \rightarrow^{H} C:=[0,1] \times\{0\} \neq \bar{C}:=\left\{0_{2}\right\}$, the latter set being the smallest compact component of $F$. Concerning the respective conic components, $D_{k}=\left\{x \in \mathbb{R}^{2}: x_{1} \geq 0,-\frac{1}{k} x_{1}+x_{2} \geq 0\right\}$ for all $k \in \mathbb{N}$ and $D=$ 
$\mathbb{R}_{+}^{2}$, we have $D_{k} \cap B_{2} \rightarrow^{H} D \cap B_{2}$. Finally, observe that $\left(C_{k}, D_{k}\right) \rightarrow^{\rho}(C, D) \neq$ $(\bar{C}, D)$, with $C+D=\bar{C}+D=F$.

In order to prove that $(\Theta, \rho)$ is a complete metric space we need the following lemmas.

Lemma 2 Let $\emptyset \neq D \subset \mathbb{R}^{n}$. Then $D$ is a closed convex cone if and only if there exists a compact convex set $Y \subset \mathbb{R}^{n}$ formed by radii of $B_{n}$ such that $D=$ cone $Y$.

Proof. If $D$ is a closed convex cone, then $Y:=D \cap B_{n}$ satisfies the required conditions.

Assume that $D=$ cone $Y$, where $Y$ is a compact convex union of radii of $B_{n}$ and $D=$ cone $Y$. We must prove that $D$ is closed.

Let $x=\lim _{k} x_{k}$, with $x_{k} \in D$ for all $k \in \mathbb{N}$. If $x=0_{n}$, we are done because $0_{n} \in Y \subset D$. Thus, the set $\left\{k \in \mathbb{N}: x_{k}=0_{n}\right\}$ is finite and we can assume w.l.o.g. that $x, x_{k} \neq 0_{n}$ for all $k \in \mathbb{N}$. Then, given $k \in \mathbb{N}$, we have $\frac{x_{k}}{\left\|x_{k}\right\|} \in Y$ because this is an extreme point of the radium of $B_{n}$ in the direction of $x_{k}$. By compactness of $Y$, we can assume w.l.o.g. the existence of $y \in Y$ such that $\frac{x_{k}}{\left\|x_{k}\right\|} \rightarrow y$. Then, since $\left\|x_{k}\right\| \rightarrow\|x\|, x=\lim _{k} x_{k}=\|x\| y \in D$.

Lemma $3\left\langle\Delta_{1}, d_{H}\right\rangle$ is a complete metric space.

Proof. Since $\mathbb{R}^{n}$ is complete for the Euclidean metric, the hyperspace $\left\langle C L\left(\mathbb{R}^{n}\right), d_{H}\right\rangle$ is complete too. So, it is sufficient to prove that $\Delta_{1}$ is a closed subset of $C L\left(\mathbb{R}^{n}\right)$.

Let $\left\{C_{k}\right\}$ be a sequence of compact convex sets such that $C_{k} \rightarrow^{H} C$. We must show that $C$ is a compact convex set.

$\left\{C_{k}\right\}$ is a Cauchy sequence it is convergent. Let $k_{0} \in \mathbb{N}$ be such that $d_{H}\left(C_{k}, C_{k_{0}}\right) \leq$ 1 for all $k \geq k_{0}$. We have $C_{k} \subset C_{k_{0}}+B_{n}$ for all $k \geq k_{0}$. Let $\mu \geq 1$ be such that $\bigcup_{k=1, \ldots, k_{0}}\left(C_{k}+B_{n}\right) \subset \mu B_{n}$. Thus, the sequence of compact convex sets $\left\{C_{k}\right\}$ is contained in $\mu B_{n}$ and we can apply Blaschke's convergence theorem: since the compact convex sets contained in a given closed ball of $\mathbb{R}^{n}$ form a compact metric space for $d_{H}$ (see, e.g., $[21, \S 4.6]$ ), $\left\{C_{k}\right\}$ contains a convergent subsequence whose limit, necessarily $C$, is a compact convex set too.

Lemma $4\left\langle\Delta_{2}, d_{H} \circ(\psi, \psi)\right\rangle$ is a complete metric space.

Proof. It is sufficient to prove that $\psi\left(\Delta_{2}\right)$ is a closed subset of the complete metric hyperspace $\left\langle C L\left(B_{n}\right), d_{H}\right\rangle$ (observe that $B_{n}$ is a closed, and so complete, subset of $\mathbb{R}^{n}$ ). 
Let $\left\{D_{k}\right\}$ be a sequence of closed convex cones such that $\left(C_{k}, D_{k} \cap B_{n}\right) \rightarrow^{H}$ $(C, Y)$ in $\left\langle C L\left(B_{n}\right), d_{H}\right\rangle$. We must show that $Y$ is the intersection of some closed convex cone $D$ with $B_{n}$. Observe that $0_{n} \in Y$ because $0_{n} \in D_{k} \cap B_{n}$ for all $k \in \mathbb{N}$ and $D_{k} \cap B_{n} \rightarrow^{P K} Y$.

Since the sequence of compact convex sets $\left\{D_{k} \cap B_{n}\right\}$ is contained in the closed ball $B_{n}$, again by Blaschke's theorem, we can assert that $D_{k} \cap B_{n} \rightarrow^{H} Y$, and $Y$ is a compact convex set.

Now we prove that the convex cone $D:=$ cone $Y=\mathbb{R}_{+} Y$ is closed, i.e., according to Lemma 2, that $Y$ is a union of radii of $B_{n}$. Let $x \in Y$ and $\lambda>0$ be such that $y:=\lambda x \in B_{n}$. If $x=0_{n}$, then $y=0_{n} \in Y$ because $0_{n} \in D_{k} \cap B_{n}$ for all $k \in \mathbb{N}$ and $D_{k} \cap B_{n} \rightarrow^{P K} Y$. Alternatively, if $x \neq 0_{n}$, then we can write $x=\lim _{k} x_{k}$, with $x_{k} \in D_{k} \cap B_{n}$ and $x_{k} \neq 0_{n}$ for all $k \in \mathbb{N}$ large enough. Let $y_{k}:=\frac{\lambda\|x\|}{\left\|x_{k}\right\|} x_{k}$, for all $k \geq k_{0}$. Since $y_{k} \in D_{k} \cap B_{n}$ for all $k \geq k_{0}$ and $y_{k} \rightarrow y, y$ belongs to the Painlevé-Kuratowski limit of $\left\{D_{k} \cap B_{n}\right\}$. Hence $\lambda x=y \in Y$. Thus, $D$ is a closed convex cone.

Finally, we show that $Y=D \cap B_{n}$. The inclusion $Y \subset D \cap B_{n}$ holds by the definition of $D$. Conversely, let $d \in D \cap B_{n}$. We have $D=$ cone $\{y \in Y:\|y\|=1\}$ because $Y$ is a union of radii of $B_{n}$. Then, there exists $y \in Y$ such that $d \in\left[0_{n}, y\right] \subset Y$. So, $D \cap B_{n} \subset Y$.

Theorem $5(\Theta, \rho)$ is a complete metric space.

Proof. It is straightforward consequence of Lemmas 3 and 4 as far as the product of complete metric spaces is a complete metric space.

\section{Stability of $\mathcal{F}$}

The next result shows that the feasible set mapping of Motzkin representations is highly stable.

Theorem 6 The set-valued mapping $\mathcal{F}: \Theta \rightrightarrows \mathbb{R}^{n}$ is closed and lsc. Moreover, $\mathcal{F}$ is usc at $\theta \in \Theta$ if and only if $\mathcal{F}(\theta)$ is either a compact set or the whole space $\mathbb{R}^{n}$.

Proof. First, we prove that $\mathcal{F}$ is closed.

Let $\left\{\theta_{k}\right\} \subset \Theta$ and $\left\{y_{k}\right\} \subset \mathbb{R}^{n}$ be such that $y_{k} \in \mathcal{F}\left(\theta_{k}\right)$ for all $k \in \mathbb{N}$, $\theta_{k} \rightarrow^{\rho} \theta \in \Theta$ and $y_{k} \rightarrow y$. let $\theta_{k}=\left(C_{k}, D_{k}\right)$, for all $k \in \mathbb{N}$, and $\theta=(C, D)$. For every $k \in \mathbb{N}$ we can write $y_{k}=c_{k}+d_{k}$, where $\left(c_{k}, d_{k}\right) \in C_{k} \times D_{k}$. 
Since $C_{k} \rightarrow^{H} C$ and the latter set is compact, the sequence $\left\{c_{k}\right\}$ is bounded and we can assume w.l.o.g. that $c_{k} \rightarrow c \in C$ (recall that $C_{k} \rightarrow{ }^{P K} C$ ). Then $d_{k} \rightarrow d:=y-c$. If $d=0_{n}, y=c \in C \subset \mathcal{F}(\theta)$ and we are done. Thus we assume $d \neq 0_{n}$ and we can also suppose w.l.o.g. that $d_{k} \neq 0_{n}$ for all $k \in \mathbb{N}$. Since $\frac{d_{k}}{\left\|d_{k}\right\|} \rightarrow \frac{d}{\|d\|}, \frac{d_{k}}{\left\|d_{k}\right\|} \in D_{k} \cap B_{n}$ for all $k \in \mathbb{N}$, and $D_{k} \cap B_{n} \rightarrow^{P K} D \cap B_{n}$, we get $\frac{d}{\|d\|} \in D$ and $d \in D$ as well. This means that $y=c+d \in C+D=\mathcal{F}(\theta)$ and so $\mathcal{F}$ is closed at $\theta$.

Now we prove that $\mathcal{F}$ is lsc.

Let $\theta=(C, D) \in \Theta$ and let $W$ be an open set in $\mathbb{R}^{n}$ such that $\mathcal{F}(\theta) \cap W \neq \emptyset$. Let $(c, d) \in C \times D$ such that $c+d \in W$. Let $W^{\prime}, W^{\prime \prime}$ be open sets in $\mathbb{R}^{n}$ such that $c \in W^{\prime}, d \in W^{\prime \prime}$ and $W^{\prime}+W^{\prime \prime} \subset W$ (selecting $\mu>0$ such that $c+d+2 \mu B_{n} \subset W$, we can take $W^{\prime}=c+\mu B_{n}$ and $\left.W^{\prime \prime}=d+\mu B_{n}\right)$. Let $0<\varepsilon_{1}<1$ be such that $c+\varepsilon_{1} B_{n} \subset W^{\prime}$ and $d+\varepsilon_{1} B_{n} \subset W^{\prime \prime}$.

For any compact convex set $C_{1}$ such that $d_{H}\left(C_{1}, C\right)<\varepsilon_{1}$, we have $C \subset$ $C_{1}+\varepsilon_{1} B_{n}$. Let $c=c_{1}+\varepsilon_{1} u$, with $c_{1} \in C_{1}$ and $u \in B_{n}$. Then

$$
c_{1}=c-\varepsilon_{1} u \in c+\varepsilon_{1} B_{n} \subset W^{\prime}, c_{1} \in C_{1} .
$$

If $D=\left\{0_{n}\right\}$ and $D_{1}$ is a closed convex cone such that $d_{H}\left(D_{1} \cap B_{n}, D \cap B_{n}\right)<$ $\varepsilon_{1}<1$, then $D_{1} \cap B_{n}=\left\{0_{n}\right\}$ (otherwise $D_{1} \cap B_{n}$ contains at least one radius of $\left.B_{n}\right)$. So, if $\theta_{1}=\left(C_{1}, D_{1}\right) \in \Theta$ satisfies $\rho\left(\theta_{1}, \theta\right)<\varepsilon_{1}$, then $\theta_{1}=\left(C_{1},\left\{0_{n}\right\}\right)$, with $d_{H}\left(C_{1}, C\right)<\varepsilon_{1}$. Thus, (6) and $0_{n} \in \varepsilon_{1} B_{n} \subset W^{\prime \prime}\left(d=0_{n}\right.$ because $\left.D=\left\{0_{n}\right\}\right)$ yield

$$
c_{1} \in C_{1} \cap W^{\prime} \subset C_{1} \cap\left(W^{\prime}+W^{\prime \prime}\right) \subset \mathcal{F}\left(\theta_{1}\right) \cap W,
$$

so that $\mathcal{F}\left(\theta_{1}\right) \cap W \neq \emptyset$.

Now we assume that $D$ contains at least one ray. Let $y \in D \cap W^{\prime \prime}, y \neq 0_{n}$. Then $\frac{y}{\|y\|} \in D \cap B_{n} \cap \frac{1}{\|y\|} W^{\prime \prime}$, so that $D \cap B_{n} \cap \frac{1}{\|y\|} W^{\prime \prime} \neq \emptyset$. The previous argument, with $D \cap B_{n}$ and $\frac{1}{\|y\|} W^{\prime \prime}$ replacing $C$ and $W^{\prime}$, respectively, shows the existence of $\varepsilon_{2}>0$ such that $C_{1} \cap \frac{1}{\|y\|} W^{\prime \prime} \neq \emptyset$, for any compact convex set $C_{1}$ such that $d_{H}\left(C_{1}, D \cap B_{n}\right)<\varepsilon_{2}$. Therefore we have $D_{1} \cap B_{n} \cap \frac{1}{\|y\|} W^{\prime \prime} \neq \emptyset$, and so $D_{1} \cap$ $W^{\prime \prime} \neq \emptyset$, when $D_{1}$ is a closed convex cone satisfying $d_{H}\left(D_{1} \cap B_{n}, D \cap B_{n}\right)<$ $\varepsilon_{2}$. In this event, there exists $d_{1}$ such that

$$
d_{1} \in W^{\prime \prime} \text { and } d_{1} \in D_{1} \text {. }
$$

If $\rho\left(\theta_{1}, \theta\right)<\min \left\{\varepsilon_{1}, \varepsilon_{2}\right\}$, from (6) and (7) we get

$$
c_{1}+d_{1} \in\left(C_{1}+D_{1}\right) \cap\left(W^{\prime}+W^{\prime \prime}\right) \subset \mathcal{F}\left(\theta_{1}\right) \cap W,
$$

so that we have again $\mathcal{F}\left(\theta_{1}\right) \cap W \neq \emptyset$ for $\theta_{1}$ sufficiently close to $\theta$. 
Finally, we characterize the upper semicontinuity of $\mathcal{F}$.

If $\mathcal{F}(\theta)=\mathbb{R}^{n}$, then $\mathcal{F}$ is trivially usc at $\theta$.

Let $\mathcal{F}(\theta)$ be bounded and let $W \subset \mathbb{R}^{n}$ be an open set such that $\mathcal{F}(\theta) \subset W$. The boundedness assumption means that $\theta=\left(C,\left\{0_{n}\right\}\right)$, where $C$ is a compact convex set. Then $\rho\left(\theta_{1}, \theta\right)<1$, with $\theta_{1}=\left(C_{1}, D_{1}\right)$, entails $D_{1}=\left\{0_{n}\right\}$.

Because $C$ is a compact set which does not intersect the closed set $\mathbb{R}^{n} \backslash W$, we can choose a scalar $0<\varepsilon<1$ such that

$$
\varepsilon<\inf \left\{\|c-x\|: c \in C, x \in \mathbb{R}^{n} \backslash W\right\}
$$

Then, if $\theta_{1}=\left(C_{1}, D_{1}\right)$ satisfies $\rho\left(\theta_{1}, \theta\right)<\varepsilon$, we have $D_{1}=\left\{0_{n}\right\}$ and $d_{H}\left(C_{1}, C\right)<$ $\varepsilon$, so that $C_{1} \subset C+\varepsilon B_{n} \subset W$. Thus $\mathcal{F}\left(\theta_{1}\right)=C_{1} \subset W$ and $\mathcal{F}$ is usc at $\theta$.

Now we assume that $\mathcal{F}(\theta)$ is an unbounded set different from $\mathbb{R}^{n}$. Let $\theta=$ $(C, D) \in \Theta$, with $D \neq\left\{0_{n}\right\}$. Consider the open set $W:=\mathcal{F}(\theta)+\operatorname{int} B_{n}=$ $C+\operatorname{int} B_{n}+D$. Obviously, $\mathcal{F}(\theta) \subset W$.

For each $\varepsilon>0$ there exists a vector $y \notin D$ such that $\|y\|=1$ and $d\left(y, D \cap B_{n}\right)<$ $\varepsilon$. Let $\theta_{1}=\left(C_{1}, D_{1}\right)$, where $C_{1}:=C$ and $D_{1}:=$ cl cone $\{D \cup\{y\}\}$. Then $D \subset D_{1}$ and $\rho\left(\theta_{1}, \theta\right)=d_{H}\left(D_{1} \cap B_{n}, D \cap B_{n}\right)<\varepsilon$. Moreover, $y \in D_{1}=$ $0^{+} \mathcal{F}\left(\theta_{1}\right)$. If $\mathcal{F}\left(\theta_{1}\right) \subset W$, then, by $W \subset \mathcal{F}(\theta)+B_{n}$, we get $y \in 0^{+} \mathcal{F}\left(\theta_{1}\right) \subset$ $0^{+}\left(\mathcal{F}(\theta)+B_{n}\right)=0^{+} \mathcal{F}(\theta)=D$ (contradiction). Hence, $\mathcal{F}$ cannot be usc at $\theta$. The proof is complete.

In the DEA motivating example in Section 1, only the compact component depends on the observed data, whereas the conic component remains fixed. The opposite situation is also conceivable. Next we show that Theorem 6 still holds in both situations.

Corollary 7 Let $C$ be a nonempty compact set and let $\mathcal{F}_{2}:\left\langle\Delta_{2}, d_{H} \circ(\psi, \psi)\right\rangle \rightrightarrows$ $\mathbb{R}^{n}$ be such that $\mathcal{F}_{2}\left(D_{1}\right)=C+D_{1}$ for all $D_{1} \in \Delta_{2}$. Then $\mathcal{F}_{2}$ is closed and lsc. Moreover, $\mathcal{F}_{2}$ is usc at $D \in \Delta_{2}$ if and only if $\mathcal{F}_{2}(D)=C+D$ is either a compact set or the whole space $\mathbb{R}^{n}$.

Proof. As an immediate consequence of Theorem $6, \mathcal{F}_{2}$ is closed and lsc. Moreover, it is usc at $D \in \Theta$ if $C+D$ is either a compact set or the whole space $\mathbb{R}^{n}$. This condition is also necessary because in the last part of the proof of Theorem 6 , assuming that $F$ is an unbounded set different from $\mathbb{R}^{n}$, we have shown that small perturbations of the conic component provoke an abrupt growth of $\mathcal{F}_{2}$.

Corollary 8 Let $D$ be a closed convex set and let $\mathcal{F}_{1}:\left\langle\Delta_{1}, d_{H}\right\rangle \rightrightarrows \mathbb{R}^{n}$ be such that $\mathcal{F}_{1}\left(C_{1}\right)=C_{1}+D$ for all $C_{1} \in \Delta_{1}$. Then, $\mathcal{F}_{1}$ is closed and lsc. Moreover, 
$\mathcal{F}_{1}$ is usc at $C \in \Delta_{1}$ if and only if $\mathcal{F}_{1}(C)=C+D$ is either a compact set or the whole space $\mathbb{R}^{n}$.

Proof. The first part is as in Corollary 7, but now we must show that small perturbations of the compact component provoke an abrupt growth of $\mathcal{F}_{1}$.

Let $F=C+D$ be an unbounded set different from $\mathbb{R}^{n}$, i.e., $\left\{0_{n}\right\} \neq D \neq \mathbb{R}^{n}$. Then there exists $\bar{d} \in$ bd $D$. By the supporting hyperplane theorem for closed convex cones, there exists $w \in \mathbb{R}^{n} \backslash\left\{0_{n}\right\}$ such that $w^{\prime} \bar{d}=0$ and $w^{\prime} d \leq 0$ for all $d \in D$. Let $\bar{c}$ be a maximizer of $w^{\prime} x$ on $C$. Then, $w^{\prime}(c+d) \leq w^{\prime} \bar{c}$ for all $c \in C$ and $d \in D$, i.e., $F \subset\left\{x \in \mathbb{R}^{n}: w^{\prime} x \leq w^{\prime} \bar{c}\right\}$ and the ray $\{\bar{c}+\lambda \bar{d}: \lambda \geq 0\} \subset$ bd $F$.

The closed set $\left\{\bar{c}+\lambda \bar{d}+\frac{1}{\lambda+1} w \in \mathbb{R}^{n}: \lambda \geq 0\right\}$ does not meet $F$ because

$$
w^{\prime}\left(\bar{c}+\lambda \bar{d}+\frac{1}{\lambda+1} w\right)=w^{\prime} \bar{c}+\frac{\|w\|^{2}}{\lambda+1}>w^{\prime} \bar{c} \forall \lambda \geq 0 .
$$

Thus, $U:=\mathbb{R}^{n} \backslash\left\{\bar{c}+\lambda \bar{d}+\frac{1}{\lambda+1} w: \lambda \geq 0\right\}$ is an open set such that $F \subset U$.

Let $0<\varepsilon<1$. Taking $\lambda=\frac{1}{\varepsilon}-1>0$, we have

$$
\bar{c}+\lambda \bar{d}+\frac{1}{\lambda+1} w \in C+D+\varepsilon w=F+\varepsilon w .
$$

So, $C+\varepsilon w \in \Delta_{1}$ satisfies $\mathcal{F}_{1}(C+\varepsilon w)=F+\varepsilon w \nsubseteq U$ despite of $d_{H}(C+\varepsilon w, C)=$ $\varepsilon\|w\| \rightarrow 0$ as $\varepsilon \searrow 0$. Thus, $\mathcal{F}_{1}$ is not usc at $C$.

\section{$5 \quad$ Stability of $\mathcal{M}$ and $\mathcal{C}$}

For the discussion of the stability properties of $\mathcal{M}$ and $\mathcal{C}$ it is convenient to consider the following partition of the parameter space $\Theta$ associated with $\theta=(C, D)$ :

$$
\begin{aligned}
& \Theta_{1}:=\left\{\left(C_{1}, D_{1}\right) \in \Theta: D_{1}=\left\{0_{n}\right\}\right\}, \\
& \Theta_{2}:=\left\{\left(C_{1}, D_{1}\right) \in \Theta: D_{1}=\mathbb{R}^{n}\right\}, \\
& \Theta_{3}:=\left\{\left(C_{1}, D_{1}\right) \in \Theta \backslash \Theta_{1}: D_{1} \text { contains no line }\right\}, \\
& \Theta_{4}:=\left\{\left(C_{1}, D_{1}\right) \in \Theta: D_{1} \text { is a proper linear subspace }\right\}, \\
& \Theta_{5}:=\left\{\left(C_{1}, D_{1}\right) \in \Theta \backslash\left(\Theta_{2} \cup \Theta_{4}\right): D_{1} \text { contains lines }\right\} .
\end{aligned}
$$

Observe that $\theta \in \Theta_{1} \cup \Theta_{2}$ if and only if $\mathcal{F}(\theta)$ is either a compact set or the 
whole space $\mathbb{R}^{n}$. Obviously, $\Theta=\cup_{i=1}^{5} \Theta_{i}, \Theta_{i}$ are disjoint sets $i=1, \ldots, 5$. $\Theta_{1}$, $\Theta_{2}, \Theta_{3}$, and $\Theta_{4}$ are open subsets of $\Theta$.

Proposition $9 \Theta_{5}$ is a nowhere dense set such that $\Theta_{5} \subset \mathrm{cl} \Theta_{3}$.

Proof. First we show that $\Theta_{5} \subset \operatorname{cl} \Theta_{3}$. Let us have a point $\theta=(C, D) \in \Theta_{5}$. Since $D$ contains lines and it is not a subspace, $D \cap(\operatorname{lin} D)^{\perp}$ is a nontrivial pointed cone and therefore its dual cone has a nonempty interior, so that there exists $y \in \mathbb{R}^{n} \backslash\left\{0^{n}\right\}$ such that $y^{\prime} d>0$ for every $d \in D \cap(\operatorname{lin} D)^{\perp}, d \neq\left\{0_{n}\right\}$. Define the pointed cone

$$
K_{\varepsilon}^{e_{n}}:=\text { cone }\left\{x \in \mathbb{R}^{n}: \sum_{i=1}^{n-1} x_{i}^{2} \leq 1, x_{n}=\varepsilon\right\} .
$$

Now, we consider the cone $K_{\varepsilon}^{y}$, which is the same, but with its symmetry axis oriented along $y$ instead of $e_{n}$. Let us consider $\theta_{\varepsilon}=\left(C, D \cap K_{\varepsilon}^{y}\right)$. Obviously, $\theta_{\varepsilon} \in \Theta_{3}$ and $\rho\left(\theta, \theta_{\varepsilon}\right) \rightarrow 0$ as $\varepsilon \searrow 0$. Thus, $\theta \in \operatorname{cl} \Theta_{3}$.

Now we suppose that $\Theta_{5}$ is a dense subset of some nonempty open set $V \subset \Theta$. Then $V \cap \Theta_{i}=\emptyset, i=1, \ldots, 4$. Therefore, $V \subset \Theta_{5}$. Taking an arbitrary $\theta \in V$, we have $\theta \in \operatorname{cl} \Theta_{3}$ (because $\theta \in \Theta_{5}$ ) and $\theta \notin \operatorname{cl} \Theta_{3}$ (because $V \cap \Theta_{3}=\emptyset$ ). Thus, $\Theta_{5}$ is a nowhere dense set.

Note that if $\theta=(C, D) \in \Theta_{4}$, then $D=0^{+} \mathcal{F}(\theta)=\operatorname{lin} \mathcal{F}(\theta)=\operatorname{lin} D$, hence $D \cap(\operatorname{lin} D)^{\perp}=\left\{0_{n}\right\} . D \cap K_{\varepsilon}^{y}=\left\{0_{n}\right\}$, where $y \neq\left\{0_{n}\right\}$ is perpendicular to $D$ and $\rho\left(\theta, \theta_{\varepsilon}\right) \nrightarrow 0 \varepsilon \searrow 0$. The only points which are close to $\theta$ in this case are the points which have as a cone a proper linear subspace of the same dimension.

The mappings $\mathcal{M}, \mathcal{C}$ and $\mathcal{F}$ coincide on $\Theta_{1}$ whereas $\mathcal{M}$ and $\mathcal{C}$ are constant (with image $\left\{0_{n}\right\}$ ) on $\Theta_{2}$, where $\mathcal{F}$ is also constant (with image $\mathbb{R}^{n}$ ). So, $\mathcal{M}$ and $\mathcal{C}$ are closed, lsc, and usc on $\Theta_{1} \cup \Theta_{2}$. The stability properties of $\mathcal{M}$ and $\mathcal{C}$ are non trivial when $\theta \in \Theta_{3} \cup \Theta_{5} \cup \Theta_{4}$. On the other hand, $\mathcal{M}$ and $\mathcal{C}$ are interesting only if $\theta \in \Theta_{3}$ because then, for $\theta_{1}$ in certain neighborhood of $\theta$, $\mathcal{M}\left(\theta_{1}\right)$ and $\mathcal{C}\left(\theta_{1}\right)$ are the efficient set of $\mathcal{F}\left(\theta_{1}\right)$ relative to the cone $0^{+} \mathcal{F}\left(\theta_{1}\right)$ and the smallest compact component of $\mathcal{F}\left(\theta_{1}\right)$, respectively. Thus we focus our attention on the case $\theta \in \Theta_{3}$. Even in the best situation that $\theta \in \Theta_{3}$, it is possible that the mappings $\mathcal{M}$ and $\mathcal{C}$ are neither upper semicontinuous, nor closed: consider $\theta=(C, D)$ and $\theta_{k}=\left(C_{k}, D_{k}\right), k \in \mathbb{N}$, as in Example 1. Then, $\mathcal{M}\left(\theta_{k}\right)=\mathcal{C}\left(\theta_{k}\right)=C$ for all $k \in \mathbb{N}$ whereas $\mathcal{M}(\theta)=\mathcal{C}(\theta)=\left\{0_{2}\right\} \nsubseteq C+$ int $B_{2}$, so that $\mathcal{M}$ and $\mathcal{C}$ are not usc at $\theta$; taking $x_{k}=(1,0) \in \mathcal{M}\left(\theta_{k}\right)=\mathcal{C}\left(\theta_{k}\right), k \in \mathbb{N}$, we conclude that $\mathcal{M}$ and $\mathcal{C}$ are not closed at $\theta$ because $x_{k} \rightarrow(1,0) \notin\left\{0_{2}\right\}$. Even more, the next example shows that $\mathcal{F}$ is not necessary closed-valued on $\Theta_{3}$. 
Example 10 Let $n=3$ and consider the set $F=C+D$, where

$C:=\operatorname{conv}\left\{\left\{x \in \mathbb{R}^{3}:\left(x_{1}-1\right)^{2}+x_{2}^{2} \leq 1, x_{3}=1\right\} \cup\left\{0_{3}\right\}\right\}$ and $D:=$ cone $\left\{e_{3}\right\}$.

Obviously,

$$
M(F)=\left\{x \in \mathbb{R}^{3}:\left(x_{1}-x_{3}\right)^{2}+x_{2}^{2}=x_{3}^{2}, 0 \leq x_{3} \leq 1, x_{1}^{2}+x_{2}^{2} \neq 0\right\} \cup\left\{0_{3}\right\}
$$

is not closed.

Next we show that both multifunctions are at least lower semicontinuous on $\Theta_{3}$.

Proposition $11 \mathcal{M}$ and $\mathcal{C}$ are lsc at every $\theta \in \Theta_{3}$.

Proof. Let $\theta=(C, D) \in \Theta_{3}$. We have

$\mathcal{M}\left(\theta_{1}\right)=M\left(C_{1}+D_{1}\right)=\left\{x \in C_{1}+D_{1}:\left(x-D_{1}\right) \cap\left(C_{1}+D_{1}\right)=\{x\}\right\} \subset C_{1}$

for all $\theta_{1} \in \Theta_{3}$.

First, we shall prove that the multivalued mapping $\mathcal{M}$ is lsc at $\theta$. Let us suppose the contrary, i.e., there exists an open set $W \subset \mathbb{R}^{n}$ such that $W \cap$ $M(C+D) \neq \emptyset$ and a sequence $\left\{\theta_{k}\right\} \subset \Theta$, such that $\theta_{k}=\left(C_{k}, D_{k}\right) \rightarrow \theta$ and for every $k \in \mathbb{N}, W \cap M\left(C_{k}+D_{k}\right)=\emptyset$ whereby $W \cap \operatorname{cl} M\left(C_{k}+D_{k}\right)=\emptyset$, as well. W.l.o.g., we may assume that $\left\{\theta_{k}\right\} \subset \Theta_{3}$, therefore cl conv $M\left(C_{k}+D_{k}\right) \subset C_{k}$.

Let $x \in W \cap M(C+D) \subset C$. By the Hausdorff convergence $C_{k} \rightarrow^{H} C$ there exists a sequence $\left\{x_{k}\right\}, x_{k} \in C_{k}$ for all $k \in \mathbb{N}$, such that $x_{k} \rightarrow x$. By (1), given $k \in \mathbb{N}, \mathcal{F}\left(\theta_{k}\right)=\operatorname{cl} M\left(C_{k}+D_{k}\right)+D_{k}$ hence $x_{k}=y_{k}+d_{k}$, where $y_{k} \in \operatorname{cl} M\left(C_{k}+D_{k}\right) \subset C_{k}$ and $d_{k} \in D_{k}$. By the same convergence, w.l.o.g., we can assume that $y_{k} \rightarrow y \in C$ and therefore $d_{k}=x_{k}-y_{k}$ is convergent too. Let $d_{k} \rightarrow d \in \mathbb{R}^{n}$. Obviously, $d_{k} \in(\|d\|+1) B_{n}$ for $k$ large enough. Having in mind that

$$
d_{H}\left(D \cap(\|d\|+1) B_{n}, D_{k} \cap(\|d\|+1) B_{n}\right) \rightarrow{ }^{H} 0
$$

we get that $d \in D$. We have that $d \neq\left\{0_{n}\right\}$, otherwise we have $y_{k} \rightarrow x$, with $x \in W$ and $y_{k} \notin W$ for all $k \in \mathbb{N}$, which is a contradiction. Then

$$
x \neq x-d=y \in(x-D) \cap C \subset(x-D) \cap(C+D),
$$

in contradiction with

$$
x \in M(C+D)=\{z \in C+D:(z-D) \cap(C+D)=\{z\}\},
$$

and this contradiction shows that $\mathcal{M}$ is lsc at $\theta$. 
Now, by the well known theorem on the convex hull mapping ([15]), we get that $\operatorname{conv} \mathcal{M}$ is lsc at $\theta$, so that $\mathcal{C}=\operatorname{cl} \operatorname{conv} \mathcal{M}$ is lsc at $\theta$ too.

The next example shows that $\mathcal{M}$ and $\mathcal{C}$ can be highly unstable on $\Theta_{2} \cup \Theta_{4} \cup \Theta_{5}$, where $\mathcal{M}(\theta)$ and $\mathcal{C}(\theta)$ cannot be interpreted as the efficient set (w.r.t. its recession cone) and the smallest compact component of $\mathcal{F}(\theta)$, respectively (actually, $\mathcal{F}(\theta)$ has no smallest compact component when $\theta \in \Theta_{2} \cup \Theta_{4} \cup \Theta_{5}$ ).

Example 12 Let $n \geq 2$ and $\theta=(C, D)$, with

$$
C=\left\{e_{1}\right\} \text { and } D=\left\{x \in \mathbb{R}^{n}: x_{n} \geq 0\right\} .
$$

Obviously, $\theta \in \Theta_{5}$ and $\mathcal{M}(\theta)=\mathcal{C}(\theta)=\left\{0_{n}\right\}$. Let us consider the pointed closed convex cones $K_{\varepsilon}^{e_{n}}, 0<\varepsilon<1$ ( $K_{\varepsilon}^{e_{n}}$ was defined in (8)). Taking $\theta_{\varepsilon}:=$ $\left(C, K_{\varepsilon}^{e_{n}}\right) \in \Theta_{3}$, we have $\mathcal{M}\left(\theta_{\varepsilon}\right)=\mathcal{C}\left(\theta_{\varepsilon}\right)=\left\{e_{1}\right\}$. Since

$$
\rho\left(\theta, \theta_{\varepsilon}\right)=\sqrt{2\left(1-\sqrt{1-\varepsilon^{2}}\right)} \rightarrow 0 \text { as } \varepsilon \searrow 0,
$$

$\mathcal{M}$ and $\mathcal{C}$ are neither upper nor lower semicontinuous, nor closed at $\theta$.

\section{Acknowledgements}

This work has been supported by MINECO of Spain, Grant MTM2011-29064C03-02.

\section{References}

[1] Beer, G.: Topologies on Closed and Closed Convex Sets. Kluwer, Dordrecht (1993)

[2] Brosowski, B.: Parametric Semi-Infinite Linear Programming I. Continuity of the feasible set and the optimal value. Math. Program. Study 21, 18-42 (1984)

[3] Cánovas, M.J., López, M.A., Parra, J.: Upper semicontinuity of the feasible set mapping for linear inequality systems. Set-Valued Anal. 10, 361-378 (2002)

[4] Chuong, T.D.: Lower semi-continuity of the Pareto solution map in quasiconvex semi-infinite vector optimization. J. Math. Anal. Appl. 388, 443-450 (2012)

[5] Cooper, W.W., Seiford, L.M., Tone, K.: Data Envelopment Analysis (2nd ed.). Springer, New York (2006)

[6] Fischer, T.: Contributions to semi-infinite linear optimization. In Brosowski, B., Martensen, E., (eds.), Approximation and Optimization in Mathematical Physics, pp. 175-199, Peter Lang, Frankfurt-am-Main (1983) 
[7] Goberna, M.A., González, E., Martínez-Legaz, J.E., Todorov, M.I.: Motzkin decomposition of closed convex sets. J. Math. Anal. Appl. 364, 209-221 (2010)

[8] Goberna, M.A., Martínez-Legaz, J.E., Todorov, M.I.: On Motzkin decomposable sets and functions. J. Math. Anal. Appl. 372, 525-537 (2010)

[9] Goberna M.A., Iusem A., Martínez-Legaz, J. E., Todorov M.I.: Motzkin decomposition of closed convex sets via truncation. J. Math. Anal. Appl. 400, $35-47$ (2013)

[10] Goberna, M.A., López, M.A.: Linear Semi-Infinite Optimization. Wiley, Chichester (1998)

[11] Goberna, M.A., López, M.A., Todorov, M.I.: Stability theory for linear inequality systems. SIAM J. Matrix Anal. Appl. 17, 730-743 (1996)

[12] Greenberg, H.J., Pierskalla, W.P.: Stability theorems for infinitely constrained mathematical programs. J. Optim. Theory Appl. 16, 409-428 (1975)

[13] Jess, A., Jongen, H.Th., Neralic, L., Stein, O.: A semi-infinite programming model in data envelopment analysis. Optimization 49, 369-385 (2001)

[14] Lucchetti, R.: Convexity and Well-Posed Problems. Springer, New York (2006)

[15] Michael, E.: Continuous selections (I), Annals of Mathematics (2nd Ser.) 63, 361-382 (1956)

[16] Mira, J.A., Mora, G.: Stability of linear inequality systems measured by the Hausdorff metric. Set-Valued Anal. 8, 253-266 (2000)

[17] Motzkin, Th.: Beiträge zur Theorie der linearen Ungleichungen. Basel: Inaugural Dissertation 73 S. (1936)

[18] Rockafellar, R.T., Wets, R.J.-B.: Variational Analysis. Springer, Berlin (1998)

[19] Tannino, T., Sawaragi, Y.: Stability of nondominated solutions in multicriteria decision making. J. Optim. Theor. Appl. 30, 229-253 (1980)

[20] Todorov, M.I.: Kuratowski convergence of the efficient sets in the parametric linear vector semi-infinite optimization. European J. Oper. Res. 94, 610-617 (1996)

[21] Van Tiel, J.: Convex Analysis: An Introductory Text. Wiley, New York (1984) 